\title{
Optimisation of cardiac resynchronization therapy in clinical practice during exercise
}

\author{
M. M. D. Molenaar • B. Oude Velthuis • M. F. Scholten • \\ J. Y. Stevenhagen • W. A. Wesselink • J. M. van Opstal
}

Published online: 3 July 2013

(C) The Author(s) 2013. This article is published with open access at Springerlink.com

\begin{abstract}
Aims Although cardiac resynchronisation therapy (CRT) is an established treatment to improve cardiac function, a significant amount of patients do not experience noticeable improvement in their cardiac function. Optimal timing of the delay between atrial and ventricular pacing pulses (AV delay) is of major importance for effective CRT treatment and this optimum may differ between resting and exercise conditions. In this study the feasibility of haemodynamic measurements by the non-invasive finger plethysmographic method (Nexfin) was used to optimise the AV delay during exercise.

Methods and results Thirty-one patients implanted with a CRT device in the last 4 years participated in the study. During rest and in exercise, stroke volume (SV) was measured using the Nexfin device for several AV delays. The optimal AV delay at rest and in exercise was determined using the least squares estimates (LSE) method. Optimisation created a clinically significant improvement in SV of $10 \%$. The relation between $\mathrm{HR}$ and the optimal $\mathrm{AV}$ delay was patient dependent. Conclusion A potential increase in SV of $10 \%$ can be achieved using Nexfin for optimisation of AV delay during exercise. A considerable number of patients showed benefit with lengthening of the AV delay during exercise.
\end{abstract}

M. M. D. Molenaar $(\bowtie) \cdot$ B. Oude Velthuis $\cdot$ M. F. Scholten •

J. Y. Stevenhagen · J. M. van Opstal

Thoraxcentrum Twente, Medisch Spectrum Twente,

Haaksbergerstraat 55, 7513 ER Enschede, the Netherlands

e-mail: m.molenaar@mst.nl

M. M. D. Molenaar

Technical Medicine, Twente University, Enschede,

the Netherlands

W. A. Wesselink

Clinical Team, Edwards Lifesciences BMEYE, Amsterdam, the Netherlands
Keywords Cardiac resynchronization therapy . Atrio-ventricular delay · Optimisation

\section{Introduction}

In a subgroup of heart failure patients, treatment by cardiac resynchronisation therapy (CRT) can be beneficial $[1,2]$ and is mostly combined with ICD therapy [3]. Although several studies have confirmed the effect of CRT on improvement in cardiac function, still one-third of patients do not experience noticeable gain from CRT $[1,2,4,5]$.

To increase the rate of responders, proper timing of pacing pulses may play a role. The proper timing of pacing pulses is of major importance for the created stroke volume and the treatment effect of CRT $[6,7]$. As CRT improves left ventricular function and increases exercise capacity, optimisation of CRT during exercise seems more meaningful than at rest. Since earlier studies show diverse outcomes regarding the optimal timing interval during higher heart rates, with perindividual variation, optimisation of the AV delay should include both measurements at rest and during exercise [8-12].

The gold standard method for general measurement of haemodynamic improvement is invasive [13]. This technique is too elaborate for routine CRT optimisation and certainly not suitable during exercise. For that reason, noninvasive methods to assess the haemodynamic state have been developed. Echocardiography is widely used but is challenging during exercise.

Recently Van Geldorp et al. [14] studied the feasibility of a non-invasive method provided by a Nexfin device (Edward Lifesciences BMEYE B.V., Amsterdam, the Netherlands) to continuously monitor beat-to-beat haemodynamics, using a finger cuff. Van Geldorp et al. concluded that this is a promising tool in the individual optimisation of CRT. The Nexfin showed a good performance on the measurement of beat-tobeat stroke volume (SV) changes, the assessment of relative 
effects induced by CRT and on the determination of the optimal AV delay. The precision of Nexfin in assessing beatto-beat SV changes was determined at around $6 \%$, which is considered precise [14-17]. In this study the Nexfin device was used to determine the optimal AV delay during different heart rates during exercise.

In our study the feasibility of a non-invasive finger plethysmographic method (Nexfin, BMEYE B.V., Amsterdam, the Netherlands) in optimisation of atrioventricular delay (AV-delay) during exercise was assessed.

\section{Materials and methods}

The Nexfin uses the volume-clamp method introduced by Czech physiologist Professor J. Peñaz [18]. Herein the cuff pressure provides an indirect measure of intra-arterial pressure. To correct the distortion in finger pressure relative to brachial artery pressure, a frequency-dependent filter is used to restore the waveform at the brachial level. The stroke volume is computed by using CO Trek, a model-based method and algorithm which uses a nonlinear, self-adaptive threeelement Windkessel model of the aortic input impedance.

All patients known in the Cardiology Department of the Medisch Spectrum Twente implanted with a CRT device in the last 4 years were included according to the inclusion criteria, as shown in Table 1. Using these criteria a patient population was selected in which confounding factors could be largely excluded.

Test protocol

\section{Preparation}

First, the rate-responsive AV delay of the CRT device is turned off, patients are seated on the exercise bicycle, connected to a 12-lead surface ECG registration system (Philips StressVue, Philips, the Netherlands) and to the Nexfin system using the proper size finger cuff and heart reference system. After application of the measurement devices, some minutes of rest are included to create steady-state conditions in the patient as well as in the measurement devices. The VV delays were not reprogrammed in order to change only one parameter (AV delay) which could influence the haemodynamic circumstances. Most of the patients had a VV delay of -20 or $-30 \mathrm{~ms}$.

\section{Rest protocol}

The optimal AV delay at rest is tested by programming AV delays starting from $40 \mathrm{~ms}$ with increments of 20 -ms steps, until either intrinsic conductance starts or until a maximum $\mathrm{AV}$ delay of $340 \mathrm{~ms}$ is reached. When the device can not be programmed in 20-ms steps (St Jude Medical Systems devices when programming AV delays $>200 \mathrm{~ms}$ ), the test is continued using 25 -ms steps. When the VV delay precludes the programming of an AV delay of $40 \mathrm{~ms}$, the first tested AV delay is set to $60 \mathrm{~ms}$ or even $80 \mathrm{~ms}$. Prior to each programming step, baseline haemodynamic parameters were collected (10 s averages). Next, the AV delay was reprogrammed and haemodynamic changes compared with baseline were measured, preventing influences of slow haemodynamic changes. Minimally two beats after reprogramming a ten-second averaging of haemodynamic parameters is performed. After this averaging, the AV delay is programmed back to the baseline setting. After a minimum of $25 \mathrm{~s}$, the preparation for the following AV delay setting starts again. After completion of this rest protocol, the optimal rest AV delay is determined as the highest SV increase from baseline to tested AV delay. This is the 'working' optimal AV delay which is used in the exercise protocol that follows. The complete protocol of the study can be found in Appendix A.

\section{Exercise protocol}

The exercise protocol consists of three stages. During the first stage, the target heart rate is set at 15 beats per minute (bpm)
Table 1 Inclusion criteria

$C R T=$ cardiac resynchronisation therapy, $E S C=$ European Society of Cardiology, $\angle B B B=$ left bundle branch block
- Intact AV conduction.

- Patients age $<80$ years

- CRT implanted on indication of LBBB and according to ESC guidelines

- Able to complete a supine bicycle exercise stress protocol

- Sinus rhythm, no patients with e.g. permanent atrial fibrillation can be included

- No prior valvular surgery.

(might alter the physiology of the heart cycle)

- CRT in situ for minimally 6 months.

(after 6 months the major part of remodelling is assumed to be completed)

- No history of irreversible ischaemia in the heart; no previous myocardial infarction or ischaemia present (irreversible ischaemia might have a major influence on heart cycle physiology and the response to CRT)

- Posterolateral placement of the left ventricular electrode 
above the resting heart rate. In stage two and three, the target heart rate is 30 and $45 \mathrm{bpm}$ higher, respectively, if tolerated by the patient. The patient is instructed to start pedalling at a comfortable cadence of 60 rotations per minute. This cadence is indicated on a screen on the handlebar of the bike. To reach the target heart rate the bicycle's resistance is adjusted. When the target HR is reached, this HR is maintained within $5 \mathrm{bpm}$ from this target rate by adjusting the bicycle's resistance and coaching the patient in the pedalling speed. The AV delays tested during the exercise protocol range from $80 \mathrm{~ms}$ shorter than the working optimal AV delay to $80 \mathrm{~ms}$ longer. After finishing the first stage, the second and subsequent third stage are performed using the same protocol. The test is ended when stage three has been completed or when the patient becomes too fatigued to continue cycling. (See also the flowchart of the test protocol in Appendix A.)

Analysis

\section{Optimal AV delay}

Baseline SV, averaged over the $10 \mathrm{~s}$ measured before AV delay programming, is subtracted from the averaged SV measured during tested AV delay. As such, the change in $\mathrm{SV}(\Delta \mathrm{SV})$ created by the tested AV delay is found.

$$
\Delta S V(m L)=\overline{S V}_{\text {tested } A V \text { delay }}(m L)-\overline{S V}_{\text {preceding baseline }}(m L)
$$

Whinnett et al. [19] demonstrated that the curve of SV response at increasing AV delays fits closely to a secondorder polynomial with a maximum. To determine the optimal $\mathrm{AV}$ delay we used the least square estimates (LSE) method (Matlab, The Mathworks, USA). This is based on minimisation of the sum of the squared residuals, with a residual being the difference between a measured value and the fitted value provided by the 2 nd order polynomial curve.

The point in which the fit over the $\Delta \mathrm{SV}$ points reaches its maximum is selected as the optimal AV delay for that stage. It is expected that the LSE creates a parabola with a top. When, however, a parabola with a minimum is created or when the calculated maximum is not within reasonably programmable $\mathrm{AV}$ delays ( $<20$ or $>$ intrinsic guidance) the stage is excluded from further analysis. Per patient the rate dependency of the optimal AV delay in the eligible stages is assessed. The mean change in optimal AV delay in milliseconds per increase in HR in beats per minute is calculated.

\section{Beneficial effect of optimisation}

The beneficial effect of optimisation is calculated as the percentage of increase in SV potentially to be created by optimisation per stage. Therefore the mean $\Delta \mathrm{SV}$ corresponding to the minimum of the polynomial fit per stage is subtracted from that corresponding to the maximum. This potential increase is divided by the measured mean SV at the minimum of the fit.

The minimum of the polynomial fit is determined considering reasonably programmable $\mathrm{AV}$ delays, the minimum being $40 \mathrm{~ms}$ and maximum being $20 \mathrm{~ms}$ below AV delay creating intrinsic guidance since in CRT continuous pacing should be preserved.

\section{Results}

Between January 2006 and December 2011, 406 patients received a CRT device. Fifty-three patients met the inclusion criteria. Twenty-two of these patients were not included in the study because of the following reasons: insufficient peripheral circulation to perform the test $(n=1)$, and not willing to perform a cycling test $(n=21)$.

The 25 male and 6 female patients all had a CRT-D device (13 Medtronic, 14 St. Jude, 4 Boston Scientific / Guidant). All patients were receiving adequate ventricular pacing with an average ventricular pacing fraction of $98 \%$. The mean time after implant was well above 6 months. Therefore it was assumed that cardiac remodelling had ended. See Table 2 for further patient characteristics.

Optimal AV delay

A total of 90 optimisation stages were performed. For all tested $\mathrm{AV}$ delays in these 90 stages the $\Delta \mathrm{SV}$ was calculated. An example of this for one stage in one patient is shown in Fig. 1.

A maximum AV delay could be determined for all tested stages in five patients. In 19 patients there were two or more stages in which a maximum AV delay could be determined.

Table 2 Patient characteristics

\begin{tabular}{lll}
\hline Sex & Male & 25 patients \\
& Female & 6 patients \\
Age & & $65.8 \pm 8.9$ year \\
CRT device & CRT-P & 0 patients \\
& CRT-D & 31 patients \\
Device brand & Medtronic & 13 patients \\
& St Jude Medical & 14 patients \\
& Boston/Guidant & 4 patients \\
EF before implant & & $26 \pm 7 \%$ \\
Time after implant & & $32.9 \pm 14.5$ months \\
Ventricular pacing & & $98 \pm 2 \%$ \\
Max stage reached & Stage 1 & 10 patients \\
& Stage 2 & 14 patients \\
& Stage 3 & 7 patients \\
\hline
\end{tabular}

$C R T=$ cardiac resynchronisation therapy, $E F=$ ejection fraction 


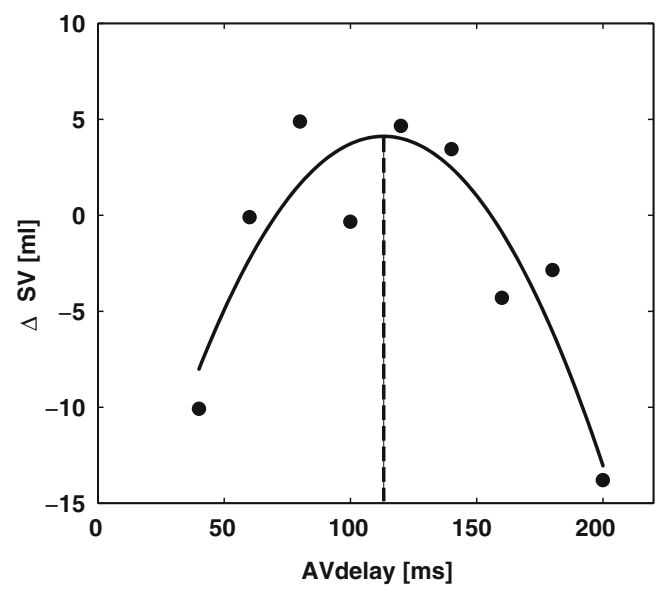

Fig. 1 Optimal AV delay calculation for one stage in one patient. The points mark the $\Delta S V$ calculations for the different AV delays. The solid curve is the individual optimisation curve created by the LSE method. The optimal AV delay is selected as the maximum of the optimisation curve (dotted line)

Using the LSE method in 55 (61\%) of the stages a maximum AV delay could be determined. See Table 3 for specifications per stage. The mean optimal AV delay was $118 \pm 32$ ms during rest stages, $121 \pm 40 \mathrm{~ms}$ during stage $1,129 \pm 30 \mathrm{~ms}$ during stage 2 and $109 \pm 31 \mathrm{~ms}$ during stage 3 . The averaged optimal AV delay over all 54 stages was $121 \pm 34 \mathrm{~ms}$. See also Table 3 .

When considering the optimal AV delay during exercise (in patients with two or more eligible stages), a shortening $(1.9 \pm 0.9 \mathrm{~ms} / \mathrm{bpm})$ of optimal AV delay was found in eight patients $(42 \%)$. In four patients $(21 \%)$ a lengthening $(2.5 \pm-$ $1.7 \mathrm{~ms} / \mathrm{bpm}$ ) of optimal AV delay was found. In four patients $(21 \%)$ the AV delay did not change with increasing heart frequency, i.e. the optimum stayed within $10 \mathrm{~ms}$. In the remaining three $(16 \%)$ patients the optimal AV delay fluctuated with increasing heart frequencies but did not solely shorten or lengthen.

\section{Beneficial effect of optimisation}

In this study a mean optimisation effect of $10 \%$ was found. In 12 stages a potential increase in stroke volume between 0 and $5 \%$ was observed, in 21 stages an effect between 5 and $10 \%$.

Table 3 Percentage of eligible stages and mean optimal atrioventricular (AV) delay per stage

\begin{tabular}{lll}
\hline Optimal AV delay & $\%$ with max & Mean \pm SD \\
\hline Rest & $61 \%$ & $118 \pm 31 \mathrm{~ms}$ \\
Stage 1 & $65 \%$ & $121 \pm 40 \mathrm{~ms}$ \\
Stage 2 & $57 \%$ & $129 \pm 29 \mathrm{~ms}$ \\
Stage 3 & $57 \%$ & $109 \pm 31 \mathrm{~ms}$ \\
Overall & $60 \%$ & $120 \pm 34 \mathrm{~ms}$ \\
\hline
\end{tabular}

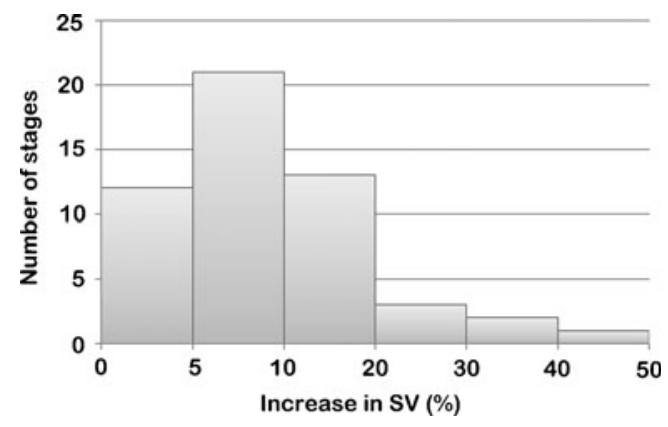

Fig. 2 Increase in SV created by optimisation

In the resting stages an effect of above $10 \%$ was observed. See also Fig. 2.

\section{Discussion}

This study was designed to assess the feasibility of Nexfin in optimisation of AV delay during exercise based on SV differences. The Nexfin device was used to determine the effect of HR increase during different levels of exercise on the optimal AV delay. Optimisation of the AV delay resulted in a $10 \%$ potential SV increase. This is a clinically relevant increase indicating that the individual patient could benefit from optimisation by Nexfin during exercise.

The optimal AV delay per stage was determined using Nexfin. Assuming the relation between $\Delta \mathrm{SV}$ and increasing $\mathrm{AV}$ delay can be described by a second order polynomial function, an optimal AV delay could be determined in $61 \%$ of the stages. The other stages showed a fit with a minimum or a fit with a maximum, which was not within reasonably programmable $\mathrm{AV}$ delays ( $<20$ or $>$ intrinsic guidance).

The mean optimal AV delay found at rest was $118 \mathrm{~ms}$. This indicates that for some patients, the typical default settings (120 ms) can be considered optimal. However, a large standard deviation in optimal AV delay was found. Since it cannot be predicted for whom the out of the box settings will be optimal, this is a strong indication that the AV delay should be optimised individually in every patient with CRT.

In $42 \%$ of the patients eligible for analysis, a shortening in optimal AV delay was found for increasing heart rates, whereas $21 \%$ showed a lengthening in optimal AV delay. These results are in accordance with the highly diverse outcomes in earlier studies [8-12]. The use of different methods for measurement of SV has been suggested as a possible explanation for this diversity. Another explanation, which our study supports, could be the patient dependency in rate responsive optimal AV delay.

In currently available CRT devices the AV delay can only be programmed to a fixed rate or to dynamic shortening, while lengthening of the AV delay during CRT is not an option. This study, and the earlier mentioned study by Scharf [9], indicated a potential benefit of lengthening of the AV 
delay for a certain patient group. Because of earlier mentioned conflicting evidence on rate adaptive $\mathrm{AV}$ delay in the literature, it is currently advised that the rate responsive AV delay should be inactivated by default $[12,20]$. However programming rate responsive $\mathrm{AV}$ delay $\mathrm{ON}$ was encouraged in the MIRACLE trials [5]. A recent pilot study by Shanmugam [21] showed that programming rate adaptive $\mathrm{AV}$ delay $\mathrm{ON}$ improves exercise capacity and advised to revise the debate regarding rate adaptive AV delay.

Only a few other devices provide continuous and noninvasive stroke volume during exercise. The Finometer (Finapress Medical Systems, Amsterdam, the Netherlands) also uses the volume clamping and a pulse contour analysis method to determine stroke volume. However, this device needs frequent calibration with an upper arm cuff. The Nicom system (Cheetah Medical, Vancouver, USA) uses bioreactance to measure stroke volume. These measurements have been found feasible and reliable for AV delay optimisation. However, this has not yet been tested during exercise where this may be a challenge due to the system's vulnerability to motion artifacts [22].

\section{Limitations}

The first limitation is that only 6 female patients were included in the study. However, women appear to be generally underrepresented in patients who receive ICDs and CRT devices [23]. Secondly, the VV delay was deliberately not reprogrammed to assess the effect of changing the AV delay only. Adapting the VV delay as well could have resulted in an even higher increase of SV. And a third limitation is that the study only describes the acute haemodynamic effects; long-term follow-up data will become available next year.

\section{Conclusion}

This study is a first step in clinical application of the Nexfin in AV delay optimisation during exercise. In every patient $\mathrm{AV}$ delay at rest and during exercise should be optimised individually. A potential increase in SV of $10 \%$ can be achieved using Nexfin for optimisation of AV delay during exercise while a considerable number of patients showed benefit with lengthening of the AV delay during exercise.

Disclosure Wilbert Wesselink is an employee of Edwards Lifesciences BMEYE, Amsterdam, the Netherlands.

Funding None.

\section{Conflict of interests None declared.}

Open Access This article is distributed under the terms of the Creative Commons Attribution License which permits any use, distribution, and reproduction in any medium, provided the original author(s) and the source are credited.

\section{Appendix A}

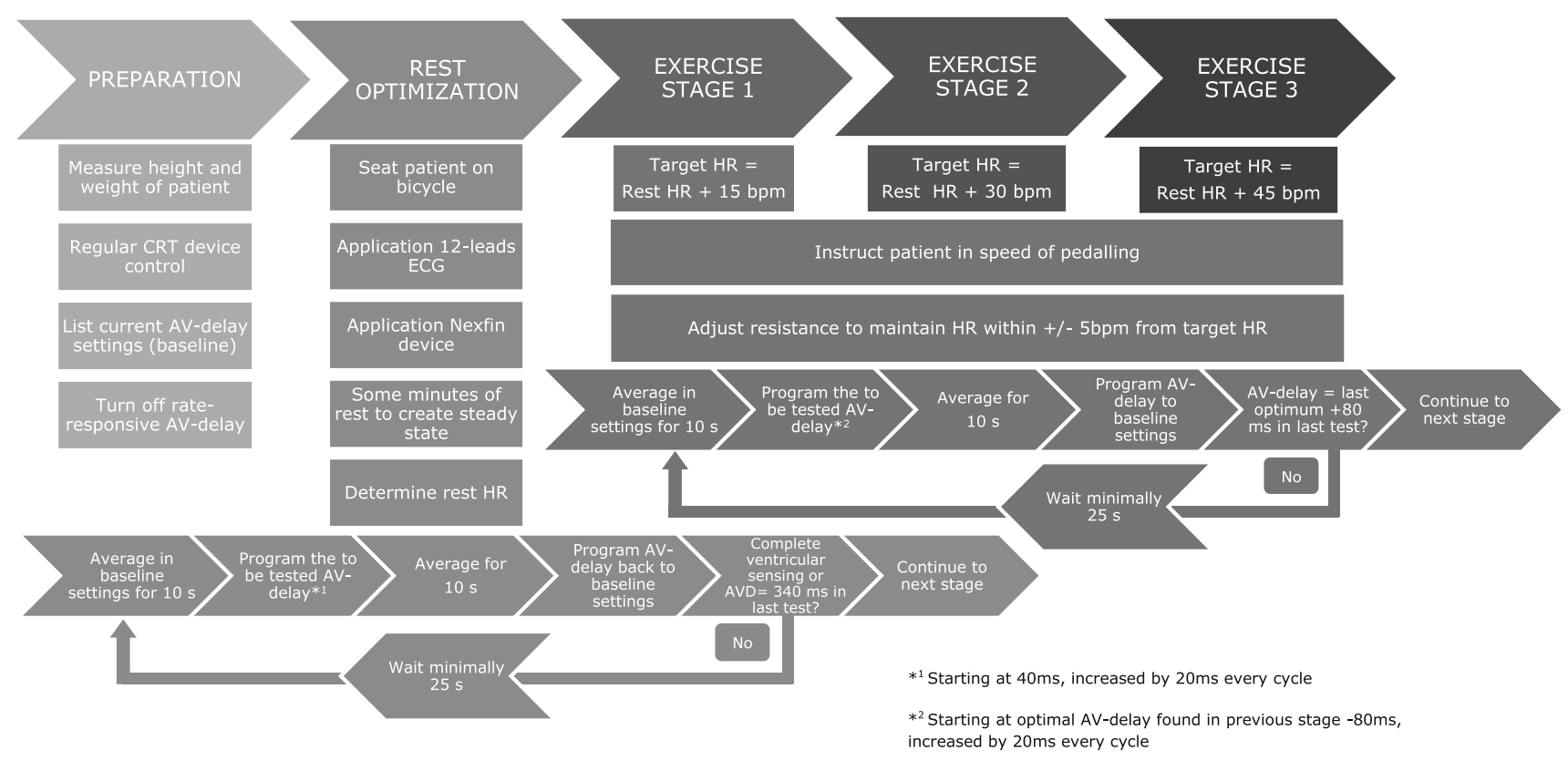




\section{References}

1. Bristow MR, Saxon LA, Boehmer J, et al. Cardiac-resynchronization therapy with or without an implantable defibrillator in advanced chronic heart failure. N Engl J Med. 2004;350:2140-50. doi:10.1056/NEJMoa032423.

2. Cleland JGF, Daubert J-C, Erdmann E, et al. Longer-term effects of cardiac resynchronization therapy on mortality in heart failure [the CArdiac REsynchronization-Heart Failure (CARE-HF) trial extension phase]. Eur Heart J. 2006;27:1928-32. doi:10.1093/eurheartj/ eh1099.

3. Smith T, Van Dessel PF, Theuns DAMJ, et al. Health care utilisation after defibrillator implantation for primary prevention according to the guidelines in 2 Dutch academic medical centres. Neth Heart J. 2011;19:405-11. doi:10.1007/s12471-011-0176-3.

4. Linde C, Leclercq C, Rex S, et al. Long-term benefits of biventricular pacing in congestive heart failure: results from the MUltisite STimulation in cardiomyopathy (MUSTIC) study. J Am Coll Cardiol. 2002;40:111-8.

5. Abraham WT, Fisher WG, Smith AL, et al. Cardiac resynchronization in chronic heart failure. N Engl J Med. 2002;346:1845-53. doi:10.1056/NEJMoa013168.

6. Auricchio A, Stellbrink C, Block M, et al. Effect of pacing chamber and atrioventricular delay on acute systolic function of paced patients with congestive heart failure. The pacing therapies for congestive heart failure study group. The guidant congestive heart failure research group. Circulation. 1999;99:2993-3001.

7. Auricchio A, Ding J, Spinelli JC, et al. Cardiac resynchronization therapy restores optimal atrioventricular mechanical timing in heart failure patients with ventricular conduction delay. J Am Coll Cardiol. 2002;39:1163-9.

8. Sun JP, Lee AP-W, Grimm RA, et al. Optimisation of atrioventricular delay during exercise improves cardiac output in patients stabilised with cardiac resynchronisation therapy. Heart. 2011. doi:10.1136/heartjnl-2011-300278.

9. Scharf C, Li P, Muntwyler J, et al. Rate-dependent AV delay optimization in cardiac resynchronization therapy. Pacing Clin Electrophysiol. 2005;28:279-84. doi:10.1111/j.1540-8159.2005.40054.x.

10. Ståhlberg M, Damgaard M, Norsk P, et al. Cardiac output response to changes of the atrioventricular delay in different body positions and during exercise in patients receiving cardiac resynchronization therapy. Europace. 2009;11:1160-7. doi:10.1093/europace/eup173.

11. Prochnau D, Forberg T, Kühnert H, et al. Optimization of the atrioventricular delay during cardiac resynchronization therapy using a device for non-invasive measurement of cardiac index at rest and during exercise. Europace. 2011. doi:10.1093/europace/eur296.
12. Melzer C, Bondke H, Körber T, et al. Should we use the rateadaptive AV delay in cardiac resynchronization therapy-pacing? Europace. 2008;10:53-8. doi:10.1093/europace/eum257.

13. Pugsley J, Lerner AB. Cardiac output monitoring: is there a gold standard and how do the newer technologies compare? Semin Cardiothorac Vasc Anesth. 2010;14:274-82. doi:10.1177/ 1089253210386386.

14. Van Geldorp IE, Delhaas T, Hermans B, et al. Comparison of a noninvasive arterial pulse contour technique and echo Doppler aorta velocity-time integral on stroke volume changes in optimization of cardiac resynchronization therapy. Europace. 2011;13:87-95. doi:10.1093/europace/euq348.

15. De Wilde RBP, Geerts BF, Cui J, et al. Performance of three minimally invasive cardiac output monitoring systems. Anaesthesia. 2009;64:762-9. doi:10.1111/j.1365-2044.2009.05934.x.

16. De Wilde RBP, Schreuder JJ, Van den Berg PCM, et al. An evaluation of cardiac output by five arterial pulse contour techniques during cardiac surgery. Anaesthesia. 2007;62:760-8. doi:10.1111/ j.1365-2044.2007.05135.x.

17. Van Lieshout JJ, Toska K, Van Lieshout EJ, et al. Beat-to-beat noninvasive stroke volume from arterial pressure and Doppler ultrasound. Eur J Appl Physiol. 2003;90:131-7. doi:10.1007/ s00421-003-0901-8.

18. Peñaz J. Photoelectric measurement of blood pressure, volume and flow in the finger. Dresden. 1973 p. 104.

19. Whinnett ZI, Davies JER, Willson K, et al. Haemodynamic effects of changes in atrioventricular and interventricular delay in cardiac resynchronisation therapy show a consistent pattern: analysis of shape, magnitude and relative importance of atrioventricular and interventricular delay. Heart. 2006;92:1628-34. doi:10.1136/ hrt.2005.080721.

20. Mokrani B, Lafitte S, Deplagne A, et al. Echocardiographic study of the optimal atrioventricular delay at rest and during exercise in recipients of cardiac resynchronization therapy systems. Hear Rhythm. 2009;6:972-7. doi:10.1016/j.hrthm.2009.03.023.

21. Shanmugam N, Prada-Delgado O, Campos AG, et al. Rate-adaptive AV delay and exercise performance following cardiac resynchronization therapy. Hear Rhythm. 2012;9:1815-21. doi:10.1016/j.hrthm.2012.07.001.

22. Khan FZ, Virdee MS, Pugh PJ, et al. Non-invasive cardiac output measurements based on bioreactance for optimization of atrio- and interventricular delays. Europace. 2009;11:1666-74. doi:10.1093/ europace/eup358.

23. Ghani A, Maas AHEM, Delnoy PPHM, et al. Sex-based differences in cardiac arrhythmias, ICD utilisation and cardiac resynchronisation therapy. Neth Heart J. 2011;19:35-40. doi:10.1007/s12471-0100050-8. 